Research article

\title{
Assessment of Equine Autoimmune Thrombocytopenia (EAT) by flow cytometry Rafael Nunez ${ }^{* 1,4}$, M. Alice Gomes-Keller ${ }^{3}$, Colin Schwarzwald ${ }^{2}$ and Karsten Feige $^{2}$
}

Address: ${ }^{1}$ Institutes of Virology, University of Zurich, Zurich, Switzerland, ${ }^{2}$ Department of Internal Medicine, University of Zurich, Zurich, Switzerland, ${ }^{3}$ Clinical Laboratory, University of Zurich, Zurich, Switzerland and ${ }^{4}$ Immunology Program, Memorial Sloan-Kettering Cancer Center, New York, USA

E-mail: Rafael Nunez* - nunezr@mskcc.org; M. Alice Gomes-Keller - magomez@vetklinik.unizh.ch; Colin Schwarzwald - schwarzw@vetklinik.unizh.ch; Karsten Feige - kfeige@vetklinik.unizh.ch

${ }^{*}$ Corresponding author

Published: 10 April 200I

BMC Blood Disorders 200 I, I:I
Received: 15 November 2000

Accepted: 10 April 200I

This article is available from: http://www.biomedcentral.com/I47I-2326/I/I

(c) 200 I Nunez et al, licensee BioMed Central Ltd.

\begin{abstract}
Rationale: Thrombocytopenia is a platelet associated process that occurs in human and animals as result of i) decreased production; ii) increased utilization; iii) increased destruction coupled to the presence of antibodies, within a process know as immune-mediated thrombocytopenia (IMT); or iv) platelet sequestration. Thus, the differentiation of the origin of IMT and the development of reliable diagnostic approaches and methodologies are important in the clarification of IMT pathogenesis. Therefore, there is a growing need in the field for easy to perform assays for assessing platelet morphological characteristics paired with detection of platelet-bound lgG.
\end{abstract}

Objectives: This study is aimed to develop and characterize a single color flow cytometric assay for detection of platelet-bound $\lg \mathrm{G}$ in horses, in combination with flow cytometric assessment of platelet morphological characteristics.

Findings: The FSC and SSC evaluation of the platelets obtained from the thrombocytopenic animals shows several distinctive features in comparison to the flow cytometric profile of platelets from healthy animals. The thrombocytopenic animals displayed i) increased number of platelets with high FSC and high SSC, ii) a significant number of those gigantic platelets had strong fluorescent signal (IgG bound), iii) very small platelets or platelet derived microparticles were found significantly enhanced in one of the thrombocytopenic horses, iv) significant numbers of these microplatelet/ microparticles/platelet-fragments still carry very high fluorescence.

Conclusions: This study describes the development and characterization of an easy to perform, inexpensive, and noninvasive single color flow cytometric assay for detection of platelet-bound lgG, in combination with flow cytometric assessment of platelet morphological characteristics in horses.

\section{Introduction}

Thrombocytopenia is a process that occurs in humans and animals as result of i) decreased bone marrow platelet production; ii) increased platelet utilization; iii) in- creased peripheral platelet destruction; or iv) platelet sequestration $[1,2]$. Thus, a differentiation of the origin of thrombocytopenia is important for defining the prognosis of the disease and the management of clinical pa- 
tients. The hallmarks of immune-mediated thrombocytopenia (IMT) are: i) enhancement of platelet destruction occurs which leads to a decrease in the number of peripheral thrombocytes and ii) the presence of antibodies associated to platelets. The antibodies, mainly of immunoglobulin $\mathrm{G}(\operatorname{Ig} G$ ) class, bind to the surface of the platelets and result in premature platelet destruction [2] IMT in horses may be primary (idiopathic thrombocytopenic purpura) [3,4], or secondary to another disorder $[1,5,6,7,8]$. IMT has been associated with equine infectious anemia virus (EIA) [8]. In this disease, there is immune complex consisting of EIA virus particles and antibodies which deposit on the platelets [5,7]. In addition, IMT featured a lack of compensatory megakaryocytopoiesis, which at the end contributed to the development of thrombocytopenia [9]. IMT has also been documented in horses with lymphosarcoma [6], and as an idiopathic disorder [1].

Important clinical signs in horses with IMT are mucosal petechiae, bleeding and mild dependent edema. The diagnosis is based on clinical signs, especially petechiae, presence of anemia, severe thrombocytopenia, increased mean platelet volume, platelet clumping, normal prothrombin time and activated partial thromboplastin time [7]. The bone marrow aspirates in IMT varied from normal to increase number of megakaryocytes [9]. In addition to the standard clinical and laboratory evaluation in cases of thrombocytopenia, it is critical to assess the presence of platelet-associated Immunoglobulin (PaIg) since patients suffering either autoimmune diseases like lupus erithematous or IMT have PaIg [10]. In humans, IMT has been assessed by flow cytometry. The flow approach has been used to detect PaIg by measurement of total IgG (including IgG contained in alpha-granules) [11], or surface IgG which is less than 1 percent of the total PaIg in normal subjects. Both fractions are usually increased in patients with IMT. However, total IgG also is increased in patients with non-immune thrombocytopenia (NIT) [12,13]. Unfortunately, the flow measurement of platelet-associated Ig only measures total Ig which may not be specific for immune-mediated disease. A test specific for immune-mediated disease, specifically in the context of drug-induced autoimmune thrombocytopenia, is not available through flow cytometry.

The current protocol for assessing PaIg in human subjects is a two color staining of fixed platelets. In brief, platelet-rich plasma is centrifuged. Then, the pelleted platelets are washed and fixed. Thereafter, a two-color flow cytometry approach is used. During the cytometric data acquisition, the platelets are gated and the mean fluorescence intensity (MFI) of the gated cells is determined. Such MFI represents a relative measure of the bound Ig. This MFI data can be reported like raw data or a more sophisticated analysis can be performed by using a standard curve, obtained after measurement the MFI for the sample compare to a mixture of fluorescent beads with varying amounts of fluorescent activity. Thus, in this approach the results are actually expressed as fluorescence equivalents, rather than the raw MFI values $[10,13]$.

The purpose of the study reported here was to develop and characterize a single color flow cytometric assay for detection of platelet-bound $\operatorname{Ig} G$ in horses in combination with flow cytometric assessment of platelet morphological characteristics.

\section{Materials and Methods \\ Cases}

Two horses with epistaxis caused by thrombocytopenia are described. In both cases primary immune mediated thrombocytopenia was diagnosed. Other disorders that could lead to thrombocytopenia were excluded based on clinical examination, endoscopy of the upper and lower respiratory system, radiographic examination of the head, hematology and blood chemistry, and determination of the coagulation parameters: i) activated partial thromboplastin time (aPTT), ii) thrombin time (TT) and iii) prothrombin time (PT). Serum protein electrophoresis, abdominal paracentesis, abdominal ultrasonography, biopsy of liver and spleen, bone marrow aspiration, serologic testing and TaqMan ${ }^{\mathrm{R}} \mathrm{PCR}^{\mathrm{a}}$ of Ehrlichiosis (E. phagocytophila genogroup), and serologic testing of EIA and Equine virus arthritis (EVA) were also performed. Horse 1 was treated with dexamethasone $(0.2 \mathrm{mg} / \mathrm{kg}$ bwt, i.v, once daily for 10 days) and did not respond to therapy. Horse 2 was treated with prednisolone $(2 \mathrm{mg} / \mathrm{kg}$ bwt, p.o., once daily for 10 days). The thrombocyte count returned within three days to a normal level.

\section{Platelet samples}

Negative-control platelets used in the development of the assay were obtained from eight healthy adult horses that underwent physical examination, and had normal hemograms, and biochemical analysis. Samples were obtained from two horses showing signs of thrombocytopenia. Horse 1 had a platelet count of 70,000 $\mu$ l. The bone marrow examination showed normal cell density, active erythropoiesis and normal megakaryocyte number. Horse 2 had a platelet count of $15,000 \mu \mathrm{l}$. Activated megakaryocytopoiesis and hyperplasia of plasmocytes were observed on the examination of the bone marrow aspirate. Both horses had normal serum protein concentration without hypergammaglobulinemia. 
Table I: Percentage of platelets-FITC positive detected within the markers (M2, M3 and M4) in normal- or thrombocytopenic-horses inside the gate RI. Underlined are the thrombocytopenic horse values that differed by more than I SD with the healthy horses

\begin{tabular}{lrrrrrrrrrrrrrr}
\hline RI & Ia & 2 & 3 & 4 & 5 & 6 & 7 & 8 & 9 & 10 & Ib & Ic & \\
\hline & & & & & & & & & & & & \\
\% of all at RI & 47.4 & 46.8 & 88.0 & 89.2 & 87.0 & 90.0 & 92.5 & 92.5 & 93.0 & 86.5 & 86.1 & 82.5 & $89.8 \pm 2.6$ \\
\% of M2 & 3.4 & 0.8 & 0.6 & 0.6 & 0.9 & 1.1 & 1.4 & 1.1 & 0.9 & 0.9 & 0.8 & 1.8 & $0.9 \pm 0.2$ \\
\% of M3 & 28.6 & 40.0 & 83.7 & 85.0 & 85.7 & 87.7 & 89.9 & 88.1 & 90.7 & 78.1 & 66.2 & 70.8 & $86.1 \pm 4.0$ \\
\% of M4 & 13.7 & 6.0 & 3.6 & 3.7 & 0.3 & 1.0 & 0.7 & 3.4 & 1.3 & 5.7 & 18.2 & 6.0 & $2.4 \pm 1.9$ \\
\hline
\end{tabular}

${ }^{*}$ Mean \pm SD of healthy horses (3-10)

Table 2: Percentage of platelets-FITC positive detected within the markers (M2, M3 and M4) in normal- or thrombocytopenic-horses inside the gate R2. Underlined are the thrombocytopenic horse values that differed by more than I SD with the healthy horses

\begin{tabular}{lrrrrrrrrrrrrrr}
\hline R2 & Ia & 2 & 3 & 4 & 5 & 6 & 7 & 8 & 9 & 10 & Ib & Ic & \\
\hline \% of all at R2 & 31.4 & 49.3 & 3.0 & 4.5 & 8.9 & 8.4 & 4.9 & 4.9 & 5.3 & 1.9 & 10.8 & 14.9 & $5.2 \pm 2.4$ \\
\% of M2\# & 4.8 & 6.4 & 1.3 & 2.0 & 2.4 & 7.5 & 3.8 & 3.4 & 3.8 & 1.2 & 2.4 & 6.7 & $3.1 \pm 2.0$ \\
\% of M3\# & 5.0 & 5.5 & 1.0 & 1.1 & 2.4 & 0.7 & 0.5 & 1.1 & 1.2 & 0.6 & 3.5 & 2.4 & $1.1 \pm .6$ \\
\% of M4 & 19.8 & 35.7 & 0.6 & 1.2 & 4.1 & 0.3 & 0.5 & 0.3 & 0.3 & 0.1 & 4.0 & 5.3 & $0.9 \pm 1.3$
\end{tabular}

${ }^{*}$ Mean \pm SD of healthy horses $(3-10) . \#: 4.8+5=9.8 \%$ (horse Ia) and $6.4+5.5=11.9 \%$ (horse 2$)$

Table 3: Percentage of platelets-FITC positive detected within the markers (M2, M3 and M4) in normal- or thrombocytopenic-horses inside the gate R3. Underlined are the thrombocytopenic horse values that differed by more than I SD with the healthy horses

\begin{tabular}{|c|c|c|c|c|c|c|c|c|c|c|c|c|c|}
\hline R3 & la & 2 & 3 & 4 & 5 & 6 & 7 & 8 & 9 & 10 & $\mathrm{Ib}$ & Ic & \\
\hline$\%$ of all at R3 & 16.9 & 2.6 & 7.9 & 5.3 & 2.7 & 2.1 & 3.2 & 2.9 & 2.6 & 10.6 & 3.6 & 3.0 & $4.7 \pm 3.0$ \\
\hline$\%$ of $M 2$ & 1.6 & 0.2 & 0.1 & 0.0 & 0.0 & 0.1 & 0.1 & 0.0 & 0.0 & 0.1 & 0.1 & 0.1 & $0.1 \pm 0.03$ \\
\hline$\%$ of $M 3$ & 1.7 & 0.6 & 2.9 & 2.1 & 1.6 & 0.8 & 1.8 & 1.4 & 1.2 & 2.9 & 1.0 & 1.5 & $1.8 \pm .75$ \\
\hline$\%$ of $\mathrm{M} 4$ & 6.8 & 1.6 & 4.6 & 3.0 & 1.0 & 1.0 & 1.2 & 1.3 & 1.4 & 5.9 & 2.1 & I.I & $2.4 \pm 1.9$ \\
\hline
\end{tabular}

*Mean \pm SD of healthy horses (3-10)

Table 4: Mean Fluorescence intensity (MFI) of platelets-FITC positive detected within the gate RI, R2 and R3 in normal- or thrombocytopenic-horses. Underlined are the thrombocytopenic horse values different by more than I SD to the healthy horses

\begin{tabular}{lrrrrrrrrrrrrrrr}
\hline MFI & Ia & 2 & 3 & 4 & 5 & 6 & 7 & 8 & 9 & 10 & Ib & Ic & \\
\hline MFI at RI & & & & & & & & & & & & & & & \\
MFI at R2 & 736 & 111 & 96 & 93 & 145 & 139 & 136 & 112 & 115 & 91 & 69 & 132 & $116 \pm 21.8$ \\
MFI at R3 & 312 & 246 & 654 & 815 & 425 & 814 & 1197 & 1026 & 841 & 1440 & 403 & 496 & $901.5 \pm 316$ \\
& 54 & 87 & 70 & 87 & 174 & 58 & 56 & 40 & 42 & 120 & $78.4 \pm 42$
\end{tabular}

\footnotetext{
*Mean \pm SD of healthy horses (3-10)
} 


\section{Laboratory procedures}

Blood was collected by jugular venipuncture into $4.5-\mathrm{ml}-$ vacutainer tubes containing $\mathrm{K}_{3}$ EDTA $^{\mathrm{b}}$ and samples were immediately processed. Of note, since all the samples were collected in similar conditions, the differences between healthy and sick horses can not be due to the presence of EDTA. Platelet-rich plasma was obtained by use of centrifugation of anticoagulated blood at $300 \mathrm{~g}$ for 5 minutes, at room temperature (RT). Platelets were pelleted by centrifugation at $300 \mathrm{~g}$ for 20 minutes, at RT. The platelet pellet was resuspended and washed 3 times in $2 \mathrm{ml}$ of PBS (o.15 M NaCl, $0.01 \mathrm{M} \mathrm{Na}_{2} \mathrm{HPO}_{4}$, and $\mathrm{pH}$ 7.4). After a third wash, the supernatant was discarded and the platelet pellet was resuspended in $250 \mu \mathrm{l}$ of goat IgG anti-equine IgG conjugated to fluorescein-5-isothio- cyanate $\left(\right.$ FITC) ${ }^{\mathrm{c}}$ diluted in PBS (dilution 1:50). The samples were incubated for 45 minutes at $37^{\circ} \mathrm{C}$ in the dark. The platelets were subsequently washed 3 times with PBS, resuspended carefully in $2 \mathrm{ml}$ of $0.5 \%$ paraformaldehyde diluted in PBS, and analyzed for fluorescence. The horses were bled for the flow cytometry analysis prior to receiving treatment. Horse 1 was followed up and 11 days later a new flow cytometry analysis of platelets was performed. This horse had just received a 10 days cycle of treatment. Moreover, Horse 1 suffered a new episode of thrombocytopenia on Dec 20, 2000. A follow up sample was taken on January 16, 2001 (approximately two weeks after therapy). Horse 2 was not available for follow up studies.
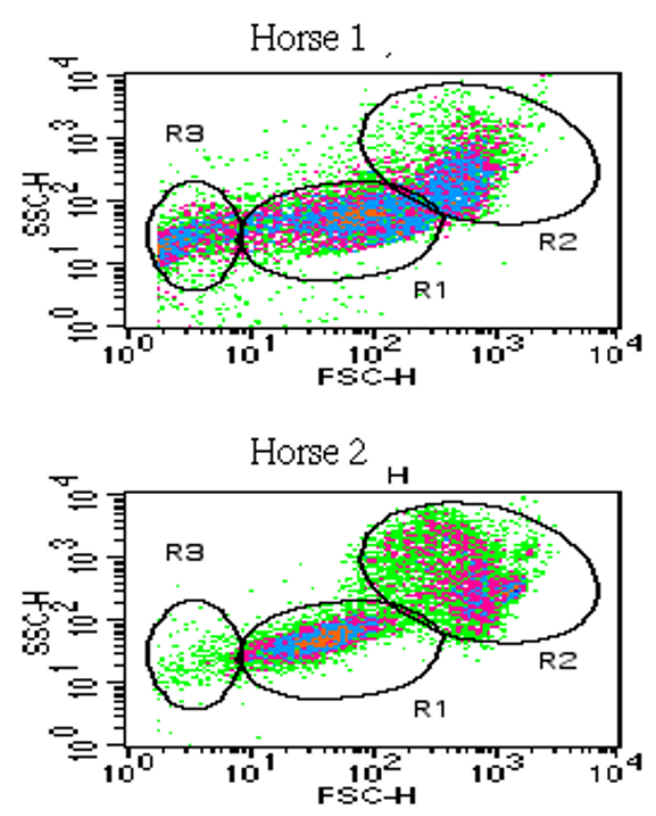

No Gate
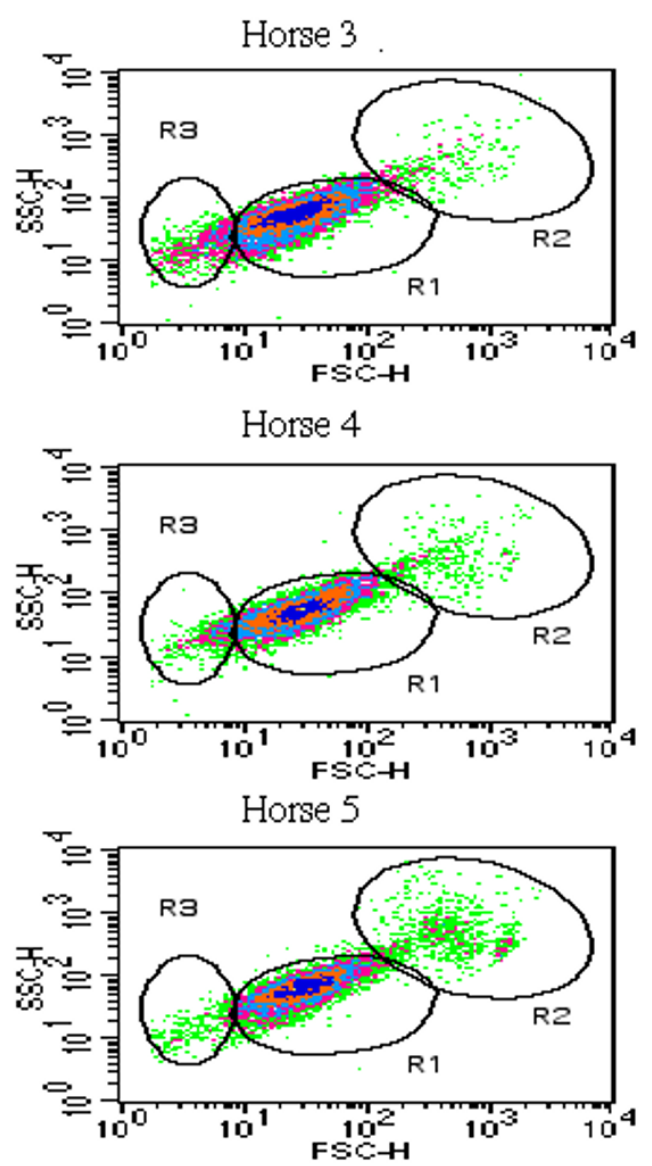

Figure I

Flow cytometric representation of morphological and fluorescent I (FL I) signal of platelets. (No gate). Density plots of ungated platelets from normal and thrombocytopenic horses. A set of panels displaying the FSC $\times$ SSC density plot of platelets derived from three healthy horses are shown in the right panels of figure Ia. The left set of panels of figure Ib represents the FSC $\times$ SSC density plots of thrombocytopenic animals. The gates RI, R2 and R3 are showed in each panel. 


\section{Flow Cytometric analysis}

The platelet morphological characteristics and Plateletbound IgG (PaIg) was determined by evaluating platelet cytometric features and fluorescence with a FACSCalibur flow cytometer ${ }^{\mathrm{d}}$. Cells $\left(10^{4}\right.$ per sample) were analyzed in a cytometer equipped for multiparametric and multicolor analysis. Forward light scatter (FSC), orthogonal scatter (SSC) and fluorescence were determined. Cells were acquired and gated by FSC and SSC. An acquisition gate (R1) containing $>90 \%$ washed normal equine platelets but excluding leukocytes and erythrocytes was drawn around the platelet cloud. This acquisition gate was used on subsequent analysis of the samples. Two additional gates were drawn in order to analyze additional groups of platelets (R2 was defined by high FSC and High SSC, and R3 was defined by low FSC and medium SSC). The gated cells were analyzed in a histogram displaying the FITC expression collected in Fluorescence 1 (green channel). FSC and SSC and the fluorescent signals were collected and storage as list mode files. Flow cytometry measurement of platelets obtained from healthy horses were also performed and used as control populations and served to determine marker borders. Using the histogram displaying the FL-1 values from healthy horse platelets, a region labeled $\mathrm{M}_{3}$, was set up to include most of the events within the characteristic sharp, narrow peak generated by normal platelets. The second region labeled M2, was generated to include everything to the right of the M3 region and represented platelets with increased fluorescence. A fourth region, labeled $\mathrm{M}_{4}$ include most of the platelets on the left of $\mathrm{M}_{3}$ and represented platelets with lower fluorescence. The region M1, spanned the three regions (M2, M3 and M4) and included about $99 \%$ of the whole platelet population within the gate. Thereafter, these regions were then applied to the histograms from thrombocytopenic horses in order to determine the percentage of platelets positive for FITC (FITC+) within the three gates (R1, R2, and R3) and within the regions (M2, M3, and M4) in the sick animals.

All the cytometric measurements of a Horse with IMT and one or two healthy control Horses were performed within a single day and under similar equipment settings in order to avoid instrument variation. Data were analyzed with CellQuest (Becton Dickinson, San Jose Ca). In total, eight healthy and two thrombocytopenic horses were evaluated. Only horse one was followed up. Negative (non labeled-) and positive (labeled platelet) samples were simultaneously assayed. Negative platelets from healthy and sick horses allowed to set up the gates (R1, R2 and R3). Statistical analysis (mean, standard deviation and graphics) was performed with Microsoft Excel on a Power Macintosh computer 8500/150.

\section{Results and Discussion}

The FSC and SSC evaluation of the platelets obtained from the thrombocytopenic animals showed quite distinctive features in comparison to the FSC and SSC profile of platelets from healthy animals. Most of the platelets from healthy animals were identified in an area located mainly between $10^{1}-10^{2}$ of the FSC axes and $10^{1}$ $10^{2}$ of the SSC axes. Then, this area was defined as gate R1 (Figure 1 (no gate), right panels). Interestingly, the thrombocytopenic animals showed two additional distinct groups of platelet clouds that prompted us to delineate them by drawing two additional gates. The new gates were labeled $\mathrm{R}_{2}$ and $\mathrm{R}_{3}$. The platelets in $\mathrm{R} 2$ displayed high FSC $\left(>10^{2}\right)$ and High SSC $\left(>10^{2}\right)$ and could represent either circulating large platelets or megakaryocytes megathrombocytes) (Figure 1 (no gate), left panels). Of note in the case of the megakaryocytes, the maximum size of the cells included in the gate R2 differed by several orders of magnitude to the cells included in the gate R1. Furthermore, The cloud observed in gate R2 for the horse 2 shows two distinctive groups of cells, (i) One with very high FSC; (ii) another with very high SSC, which suggested great internal complexity. Interestingly, horse 1 has in R2 only the cells with high FSC. Further assays including sorting of these cells and staining with antibodies could help to clarify and define the two groups of cells (Figure 1 (no gate), left panels). The platelets in $\mathrm{R}_{3}$ displayed low FSC $\left(<10^{1}\right)$ and medium $\operatorname{SSC}\left(10^{1}-10^{2}\right)$ and represent either very small platelets or platelet derived microparticles (Figure 1 (no gate), left panels). Figure 1 overall shows that the healthy animals (right panels) have the bulk of platelet population mainly located in R1 (> 86\%), while the thrombocytopenic animals (left panels) have $<48 \%$ (Figure 1 (no gate) and Table 1 , row: \% of total within R1). In sharp contrast, the healthy ones have only a minute platelet population located in R2 $(<9 \%)$, whereas the sick ones have more than $31 \%$ of the total platelet counts within the R2 gate (Figure 1 (no gate) and Table 2, row: \% of total within R2). Since platelets in R2 are characterized by high FSC and high SSC, and only the thrombocytopenic horses had significant numbers of platelets within this gate, is likely that the presence of circulating large platelets or megakaryocytes will be tightly associated with this disease. The presence of platelet aggregates could be another plausible explanation for the cloud observed in R2. However, only the thrombocytopenic animals have this cloud while the healthy ones do not. Thus, if the cloud were a complex of platelet aggregates, it should be tightly associated to the disease. Further morphological studies after sorting analysis could help to clarify this issue. In addition, one of the thrombocytopenic animals (Horse 1) showed the presence of a significant population of platelets with low FSC and normal SSC that represent either micro- 

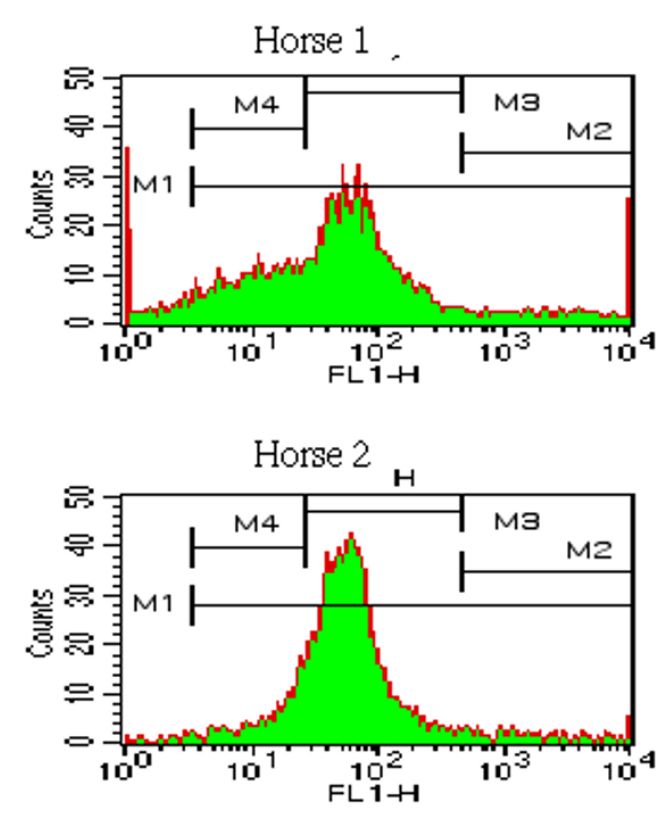

Gate R1
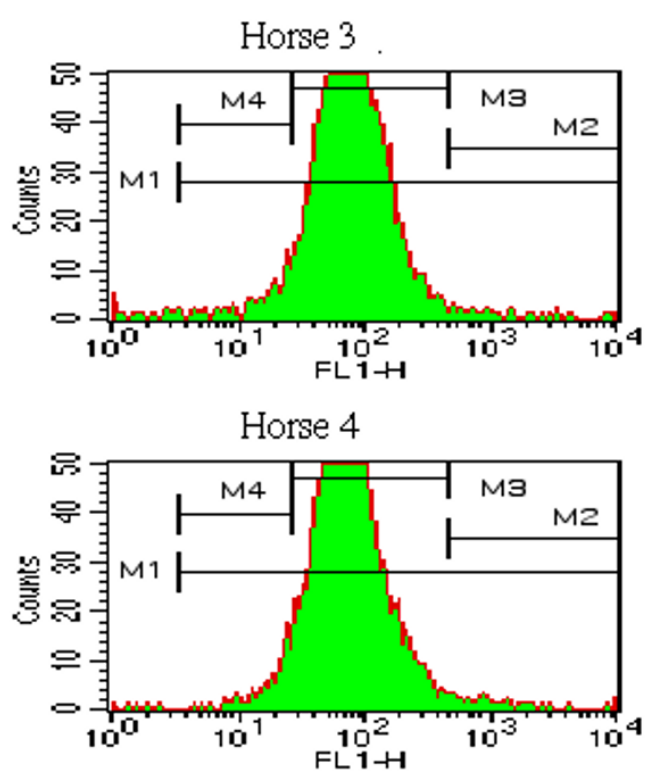

Horse 5

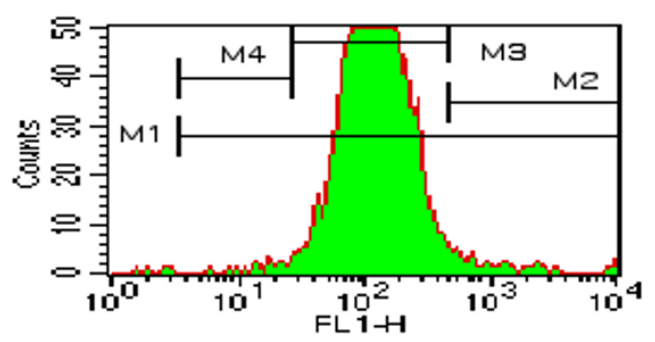

Figure 2

Flow cytometric representation of morphological and fluorescent I (FL I) signal of platelets. Histogram plots of the fluorescence signal carried by platelets delineated within gate RI.The evaluation of the presence of platelet associated IgG (Palg) was determined by performing histogram analysis of the fluorescence carried by the platelets gated into RI. Markers MI, M2, M3 and M4 were drawn within the histogram plots.

thrombocytes or platelet fragments. This platelet subset is included within the gate R3 (Figure 1 (no gate), panel: Horse 1).

The evaluation of the presence of IgG bound to platelets was also assessed by performing histogram analysis of the fluorescence carried by the platelets gated into R1 (Figure 2 (Gate R1). The platelets from healthy horses showed the characteristic sharp, narrow peak traditionally described in platelets containing alpha granules. The $\alpha$-granules of platelets contains $\operatorname{IgG}$, which is secreted from platelets in response to thrombin $[11,14]$. The techniques of platelet washing and incubation, which can cause platelet secretion of the IgG contained within the $\alpha$-granules, may be full of critical and uncontrolled variables that affecting the measurement of platelet-bound
IgG [11]. Therefore, it would be possible that after being released from the $\alpha$-granules, IgG can bind to Fc RII (medium affinity IgG receptor expressed in different cell types, including platelets) on the platelet surface, leading to misinterpretation of platelet-bound IgG measurements. Thus, this peak can be mistakenly considered as IgG bound to platelets (Figure 2 (Gate R1), right set of panels). The marker M3 determined this peak. However, the thrombocytopenic animals showed a clear reduction in the height of the peak but with widening of the base as is shown in figure $1 \mathrm{~b}$ (Figure 2 (Gate R1), left panels). Table 1 also showed that the percentage of platelets in $\mathrm{M}_{3}$ for the healthy animals was higher than $83 \%$ while in the sick ones was below $40 \%$. Within this gate, there is an increase number of platelets in the sick animals, about $6 \%$ for one horse and about $14 \%$ for the other horse that dis- 

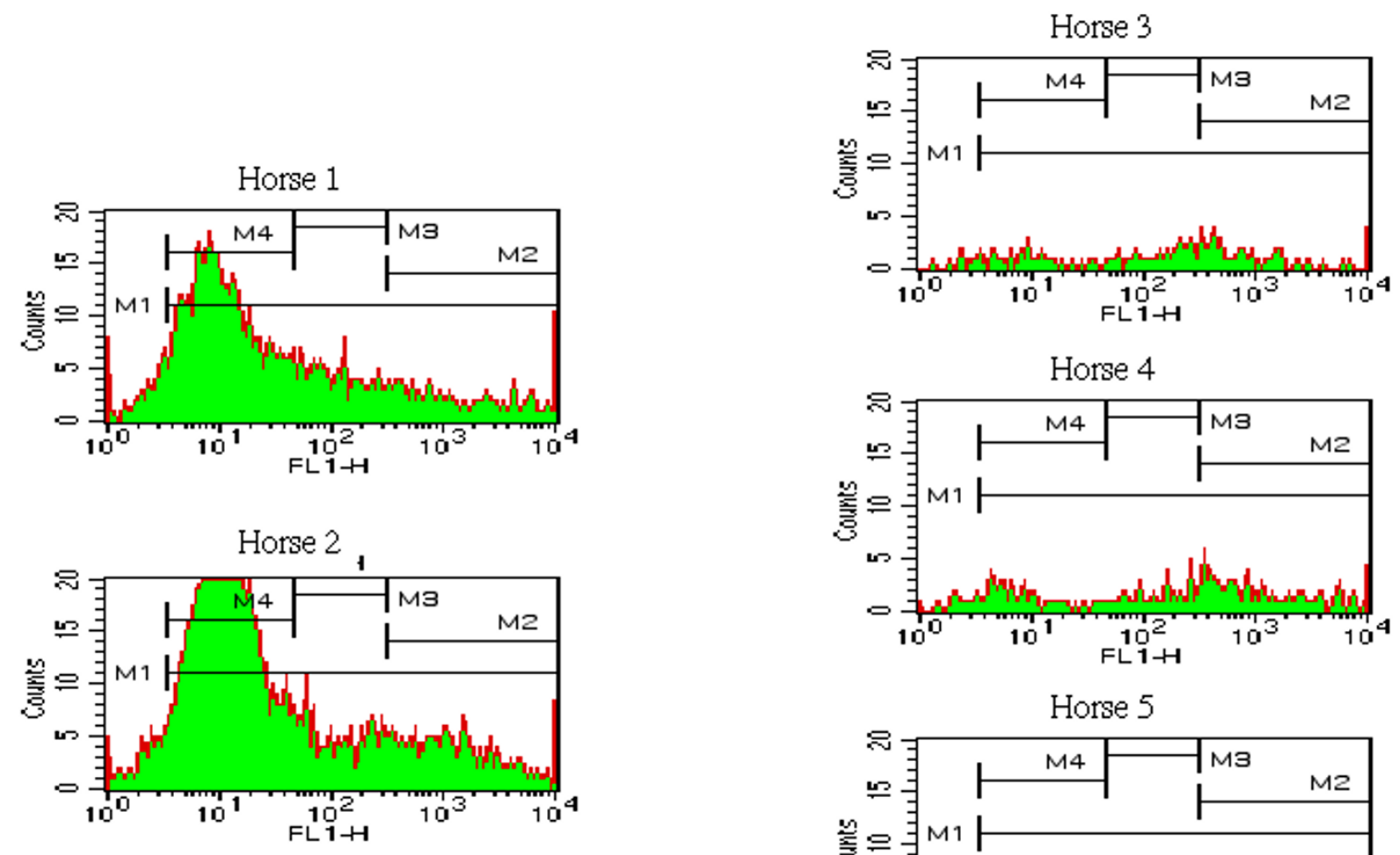

\section{Gate R2}

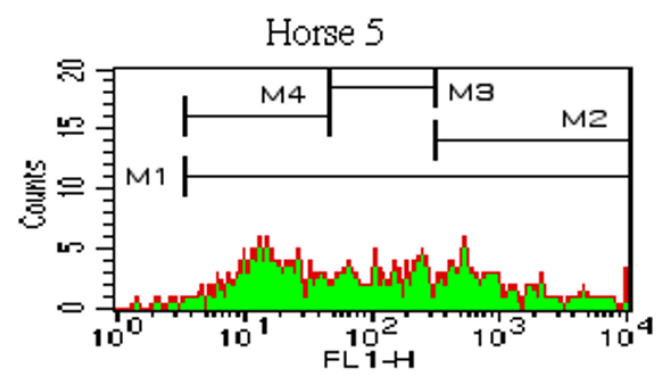

Figure 3

Flow cytometric representation of morphological and fluorescent I (FL I) signal of platelets. Histogram plots of the fluorescence signal carried by platelets delineated within gate R2. Palg was determined by performing histogram analysis of the fluorescence carried by the platelets gated into R2. Markers MI, M2, M3 and M4 are identical to the displayed in Figure 2

played lower levels of fluorescence signal on region $\mathrm{M}_{4}$ (Table 1). Such findings contribute to explain the widening in the base of the peak for the platelets in gate R1. Overall, it is clear that the thrombocytopenic animals have less platelets on the gate $\mathrm{R} 1$ and that those platelets displayed lower fluorescence signal.

The set of observations found on gate R 1 offered a sharp contrast with the findings detected on gate R2. The histogram analysis of the fluorescence displayed by the platelets gated into R2 from healthy animals (Figure 3 (Gate R2), right panels) showed that the normal animals have very few platelets with high FSC and high SSC. However, the thrombocytopenic animals displayed a quite distinctive pattern (Figure 3 (Gate R2), left panels). The profile displayed a peak in $\mathrm{M}_{4}$ that included a significant proportion of the platelets of the gate R2. However, the M4 fluorescence peak was located in the zone of low fluorescence. Of note, the thrombocytopenic animals also had a significant proportion of platelets ( $>10 \%)$ within the zone of very high intensity of fluorescence (Figure 3 (Gate R2), M2 region of left panels) and high FSC and high SSC, while the healthy ones always had at the most, $4 \%$ of platelets with these characteristics (Figure 3 (Gate R2), M2 region of right panels, and Table 2).

The R3 gate defined platelets with low FSC $\left(<10^{1}\right)$ and medium SSC $\left(10^{1}-10^{2}\right)$, which represent very small plate- 
Horse 1

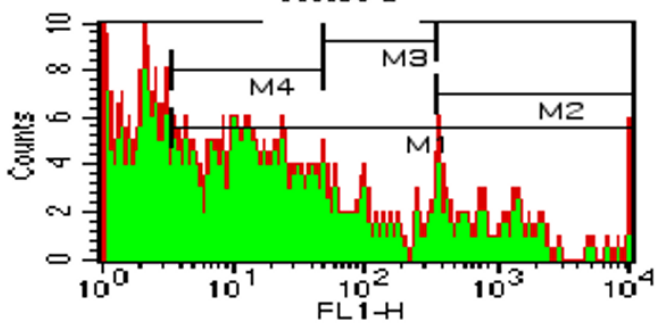

Horse 2

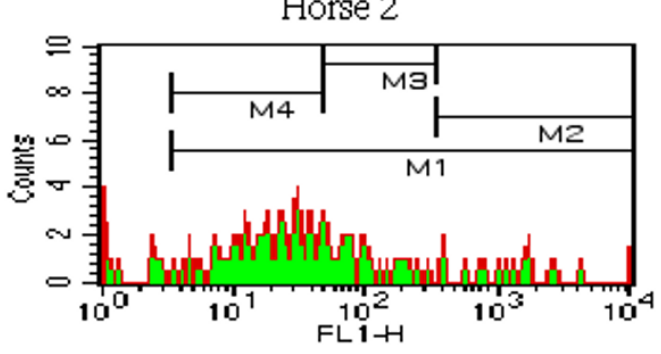

Gate R3
Horse 3

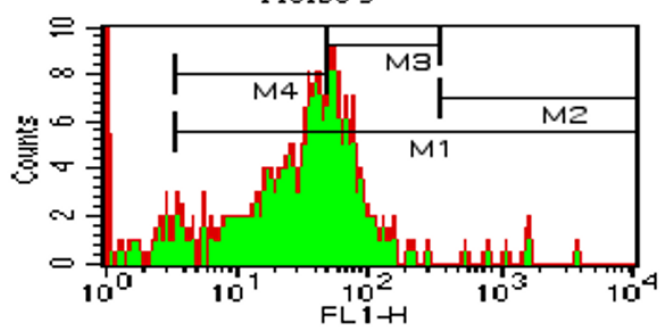

Horse 4

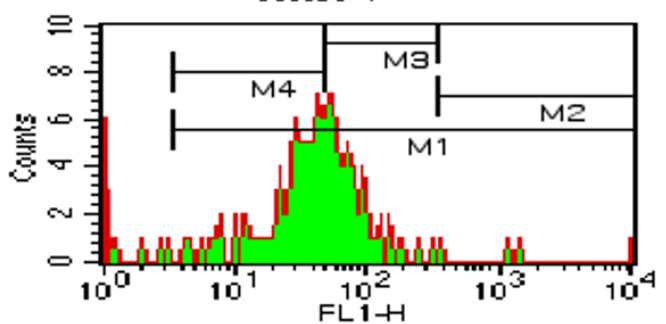

Horse 5

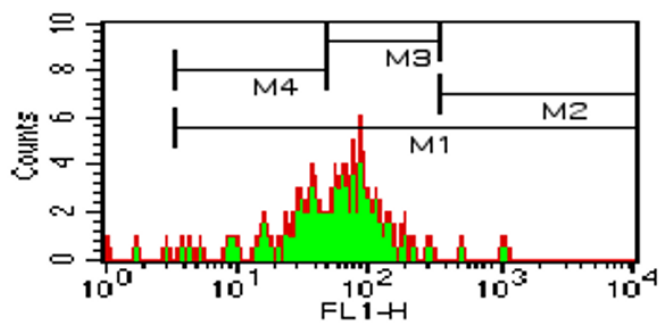

Figure 4

Flow cytometric representation of morphological and fluorescent I (FL I) signal of platelets. Histogram plots of the fluorescence signal carried by platelets delineated within gate R3. Palg was determined by performing histogram analysis of the fluorescence carried by the platelets gated into R3. Markers MI, M2, M3 and M4 are identical to the displayed in Figure 2.

lets or platelet derived microparticles (Table 3). This fraction was found significantly enhanced in one of the thrombocytopenic horses (Horse 1) as is shown in figure 1 and Table 3. Interestingly, some of these platelets still carry very high fluorescence (Figure 4 (Gate R3), Horse 1 panel-M2 region) (Table 3 and Table 4, MFI of R3). Of note, both horses with thrombocytopenia have a higher number of platelets in R2, but one horse had the higher percentage of strongly fluorescent platelets in R2 and low number of microparticles while the other one had an inverted pattern consisting of less percentage of fluorescent platelets in R2 but higher number of fluorescent microparticles (Figure 5, Table 2 and Table 4). These findings raise the possibility that the antibodies might bind to the gigantic platelets found in R2 (circulating large platelets or megakaryocytes or even platelet aggregates) and remain associated to them all the way through the pathological fragmentation process until they turn down into microparticles (Figure 5, Figure 7 and Table 3). It has been described that patients with IMT showed an increased percentage of both: microthrombocytes or platelet fragments and megathrombocytes [15,16], a finding similar to the observed with the two IMT horses described in this report.

Follow up of the thrombocytopenic horses was only feasible in horse 1. Sample 1a defines the sample obtained before treatment. Sample 1b was obtained after finishing the first cycle of treatment. It is clear that there was improvement in the flow profile since the high fluorescent microthrombocytes or platelet fragments almost disappeared and the number of megathrombocytes decreased but they did not disappear (Table 3 and 4). Further follow up showed that the number of platelets in gate R2 in- 

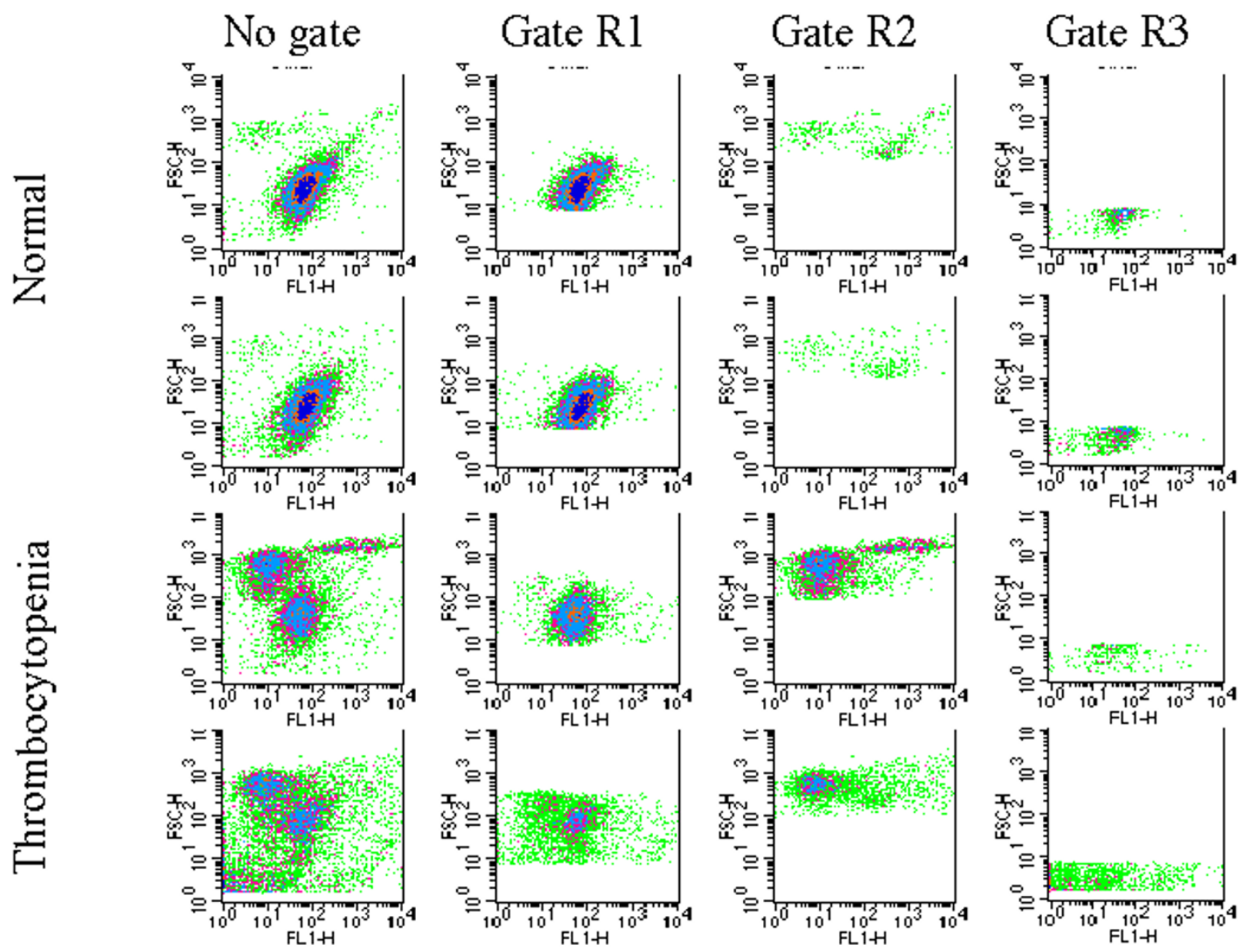

\section{Figure 5}

Flow cytometric Density plot representation of platelets from Thrombocytopenic and normal horses. The two upper rows display the set of panels (FSC $\times$ FLI plots of platelets) from two healthy horses while the two lower rows display the set of panels (FSC $\times$ FLI plots of platelets) from two thrombocytopenic horses. The corresponding density plot (FSC $\times$ FLI) within the gates are shown in the columns. No gate (external left column). Gate RI (internal left column. Gate R2 (internal right column). Gate R3 (external right column).

creased almost to normal levels (Figure 6 and 7 and table 3 and 4). For the sake of comparison, included is the protocol for analyzing and defining the gates and markers in an unstained sample of a healthy horse. There is clearly a cloud of platelets in G1 but almost nothing at gate G2 and G3 (Figure 8). Furthermore, the level of autofluorescence in unstained platelets is minimal and the signal FL1 is less than 10 in the FL-1 log scale (Figure 8).

In this study, we evaluated a flow cytometric assay for detecting platelet-bound immunoglobulin $\mathrm{G}$ in horses. In comparison to the standard approach developed by George $\mathrm{JN}$ to study human platelets $[10,13]$, this assay is easy to perform, inexpensive, and noninvasive and does not require that platelets from normal subjects be in- cluded in each assay. Together with clinical examination, laboratory tests and bone marrow evaluation, the test described in this report provides important information that may help to define the cause of the disease and the management of clinical patients and prognosis [12]. Thus, this study provides clear evidence that the antibodies (IgG isotype) are bound to abnormal platelets in horses with thrombocytopenia but the role of such antibodies in the pathogenesis of the disease remains to be addressed in further studies. However, based on the fact that the animals with thrombocytopenia displayed i) increased number of platelets with high FSC and high SSC and that ii) a significant number (9.8\% for horse 1a and $11.9 \%$ for horse 2) of those gigantic platelets displayed higher fluorescent signal (IgG bound as determined by 


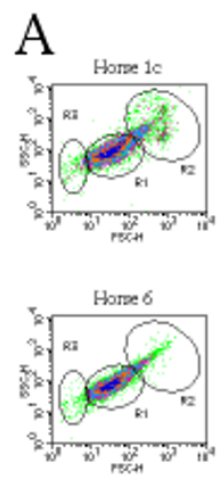

No Gate
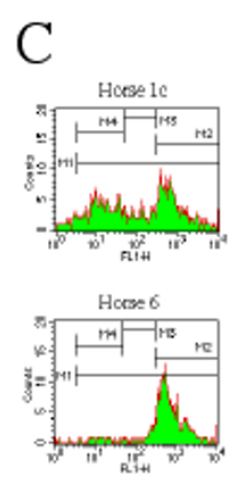

Gate R2
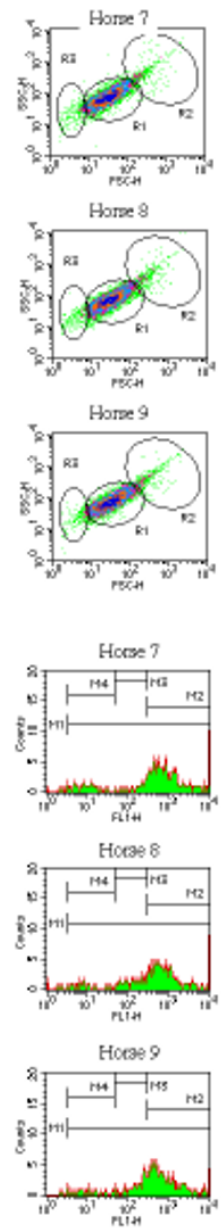

B

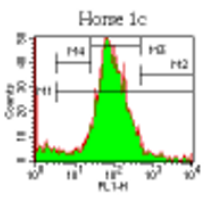

Horse 6

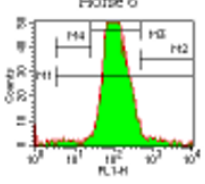

Gate Rl
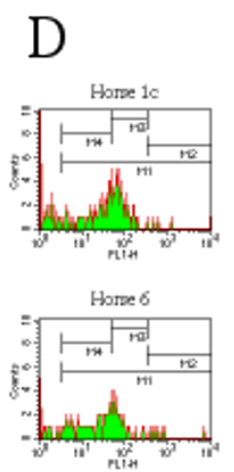

Gate R3
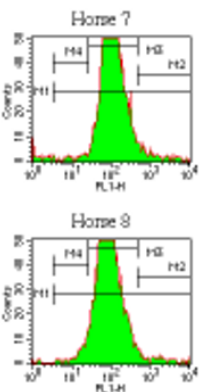

Horse 9
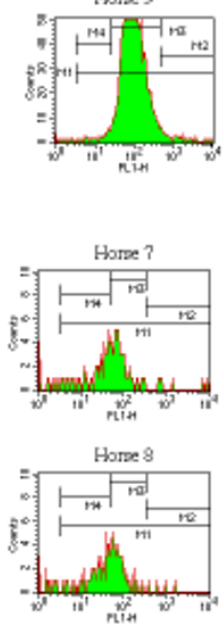

Horre 9

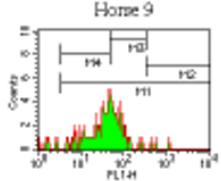

\section{Figure 6}

Flow cytometric representation of morphological and fluorescent I (FL I) signal of platelets of Horse I three weeks after new episode of thrombocytopenia and comparison with four healthy horses. Figure 6A. Density plots of ungated platelets from a thrombocytopenic and four normal horses. A set of panels displaying the FSC $\times$ SSC density plot of platelets derived from four healthy horses (Horse 6-9) are showed. The upper left panel of figure $6 \mathrm{~A}$ represents the FSC $\times$ SSC density plots of thrombocytopenic Horse Ic. The gates RI, R2 and R3 are showed in each panel. Figure 6B. Histogram plots of the fluorescence signal carried by platelets delimited within gate RI.The evaluation of the presence of platelet associated IgG (Palg) was determined by performing histogram analysis of the fluorescence carried by the platelets gated into RI. Markers MI, M2, M3 and M4 were drawn within the histogram plots. Markers are identical to the displayed in Figure 2 Figure 6C. Histogram plots of the fluorescence signal carried by platelets delimited within gate R2. Palg was determined by performing histogram analysis of the fluorescence carried by the platelets gated into R2. Markers MI, M2, M3 and M4 are identical to the displayed in Figure 3 Figure 6D. Histogram plots of the fluorescence signal carried by platelets delimited within gate R3. Palg was determined by performing histogram analysis of the fluorescence carried by the platelets gated into R3. Markers MI, M2, M3 and M4 are identical to the displayed in Figure 4.

adding the \% of platelets in M2 and M3-Table 2), it is tempting to hypothesize that such antibodies play a significant role in the earlier stages of the platelet maturation/production and may contribute to the generation of microparticles in later stages of the disease $[17,18]$. Further investigations with human subjects affected of AIT remain to be performed in order to expand the scope and validate within other mammals the findings of this study. 


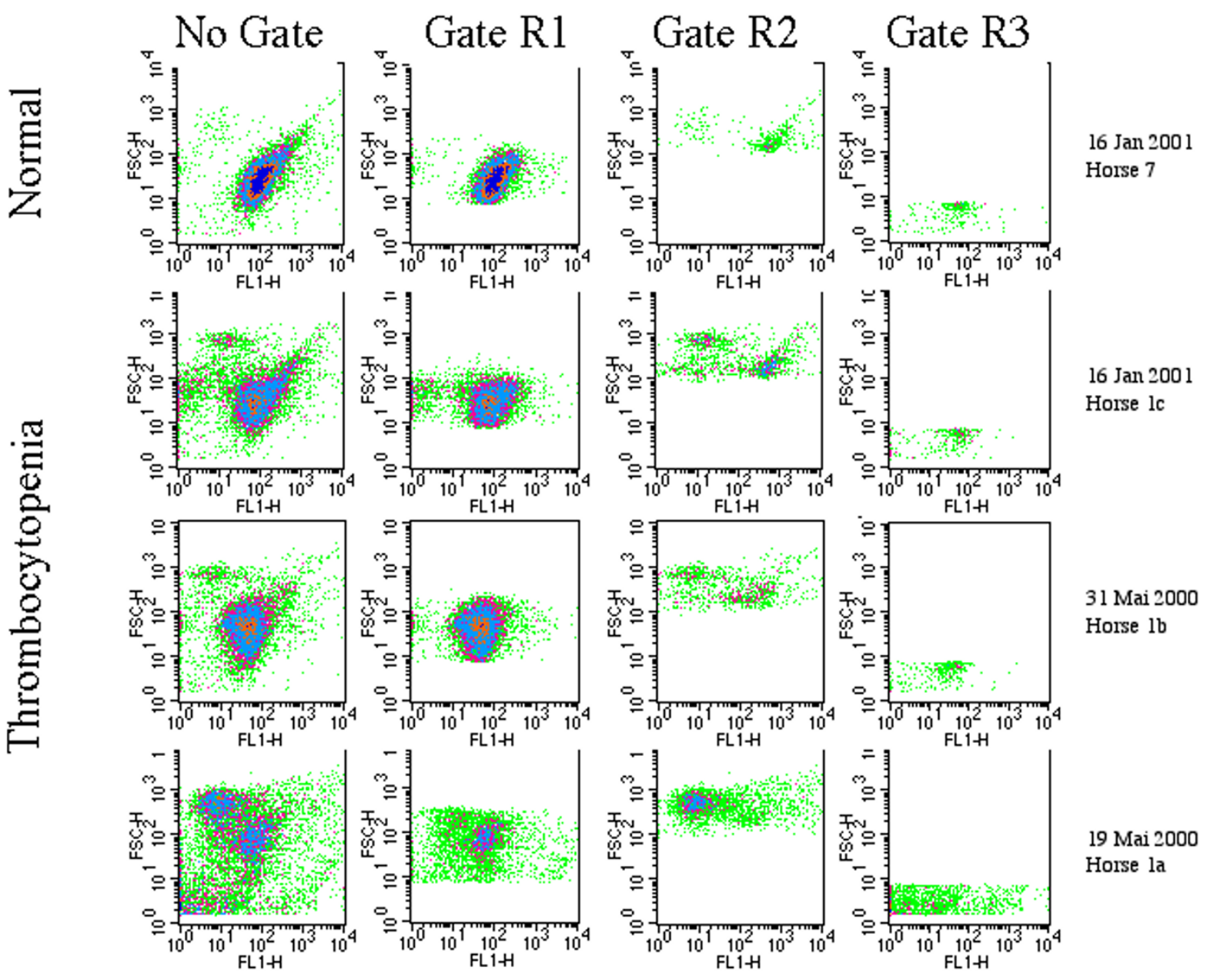

Figure 7

Flow cytometric Density plot representation of platelets from a Thrombocytopenic horse before treatment and during follow up. The three lower rows display the set of panels (FSC $\times$ FLI plots of platelets) from a thrombocytopenic horse. The lower row represents the sample labeled Horse la. This sample was taken prior to treatment, while the two middle rows display the set of panels (FSC $\times$ FLI plots of platelets) from the follow up. The date is listed. A healthy horse is displayed in the upper row. The corresponding density plot (FSC $\times$ FLI) within the gates are showed in the columns. No Gate (external left column). Gate RI (internal left column). Gate R2 (internal right column). Gate R3 (external right column). 

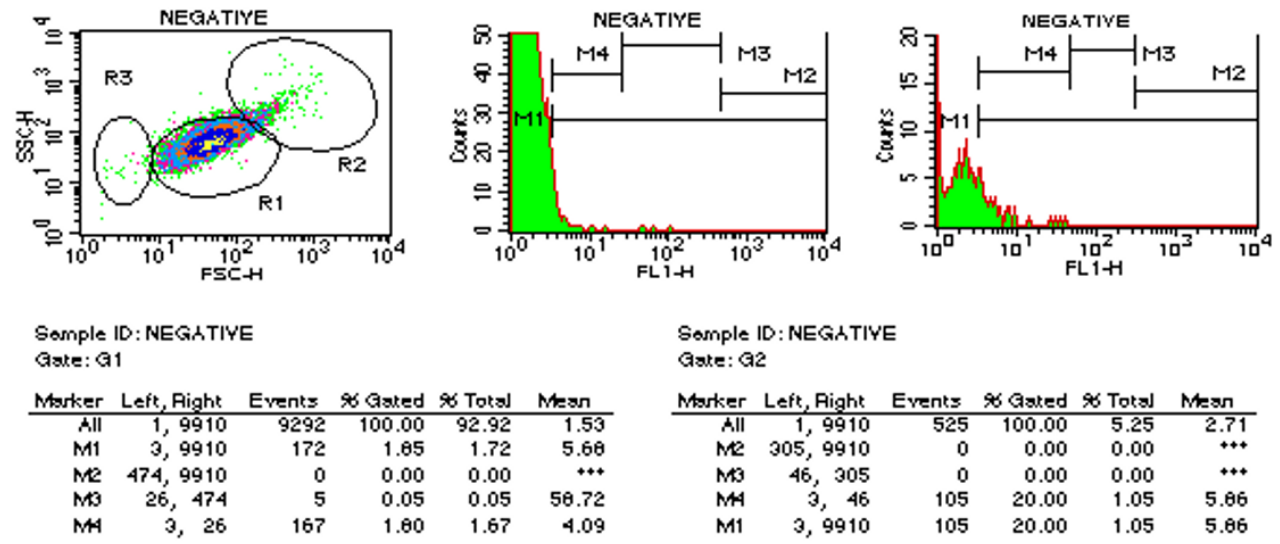

Somple ID : NEGATIYE

Gate: G2

\begin{tabular}{rrrrrr} 
Morker & Left, Pight & Events & \& Garted & \& Totzl & Mesn \\
\hline All & 1,9910 & 525 & 100.00 & 5.25 & 2.71 \\
M2 & 305,9910 & 0 & 0.00 & 0.00 & $++*$ \\
M3 & 46,305 & 0 & 0.00 & 0.00 & $+*+$ \\
M4 & 3,46 & 105 & 20.00 & 1.05 & 5.86 \\
M1 & 3,9910 & 105 & 20.00 & 1.05 & 5.86
\end{tabular}

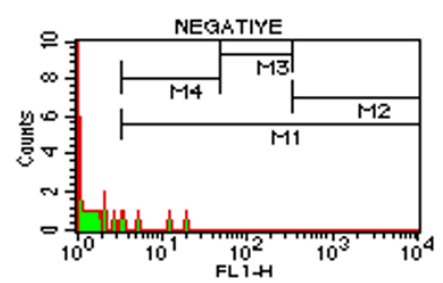

Somple ID : NEGATIYE Gate: 93

\begin{tabular}{|c|c|c|c|c|c|}
\hline Morker & Left, Right & Events & * Gated & $\$$ Total & $M=s \pi$ \\
\hline All & 1,9910 & 42 & 100.00 & 0.42 & 2.00 \\
\hline M1 & 3,9910 & 4 & 9.52 & 0.04 & 9.60 \\
\hline$M-4$ & 3,46 & 4 & 9.52 & 0.04 & 9.60 \\
\hline Mo & 46,331 & 0 & 0.00 & 0.00 & $+*+$ \\
\hline$M 2$ & 331,9910 & 0 & 0.00 & 0.00 & +*++ \\
\hline
\end{tabular}
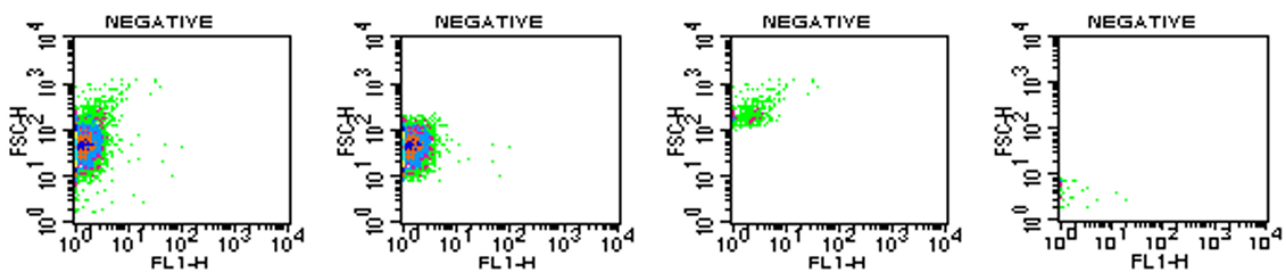

\section{Figure 8}

Protocol for the flow cytometric representation of morphological and fluorescent I (FL I) signal and statistical analysis of platelets before staining (Negative sample) of a healthy Horse. Density plot (FSC $\times$ SSC) of unstained and ungated platelets from a normal horse is displayed the upper left row. Histogram plots of the fluorescence signal carried by unstained platelets delineated within gate RI, R2 and R3 are displayed in the upper and middle rows. Marker MI, M2, M3 and M4 are identical to previous figures. Statistical analysis of each gate is displayed in middle rows. The lower row displays the set of four panels (FSC $\times$ FLI plots of platelets). No Gate (external left panel). Gate RI (internal left panel). Gate R2 (internal right panel). Gate R3 (external right panel)

\section{Acknowledgements}

Rafael Nunez and Alice Gomes made equal contribution to this study and should be considered first authors. The work was partly supported by the Swiss National Science Foundation (grant 31-45903). We express our gratitude to Drs. M. Ackermann and H. Lutz for their support to this study. also we thank D. Domingo (Memorial SloanKettering Cancer Center) for her review of the manuscript.

\section{Footnote}

a PE Biosystems, Foster City, CA b Becton Dickinson, Plymouth, UK

c Organon Teknika Corporation, CAPPEL Research Products, Durham, NC.

d FACSCalibur, Becton Dickinson Immunocytometry Systems, Mountain View, Calif.

\section{References}

I. Sellon DC, Levine J, Millikin E, Palmer K, Grindem C, Covington P: Thrombocytopenia in horses: 35 cases (1989-1994). J Vet Intern Med 1996, 10:|27-3|

2. Newman PJ, Derbes RS, Aster RH: The human platelet alloantigens, PIAI and PIA2, are associated with a leucine33/ proline 33 amino acid polymorphism in membrane glycopro- 
tein IIIa, and are distinguishable by DNA typing. / Clin Invest 1989, 83:|778-8|

3. Byars TD, Greene CE: Idiopathic thrombocytopenic purpura in the horse. J Am Vet Med Assoc 1982, I 80:1422-4

4. Larson VL, Perman V, Stevens JB: Idiopathic thrombocytopenic purpura in two horses. J Am Vet Med Assoc 1983, 183:328-30

5. Clabough DL, Gebhard D, Flaherty MT, Whetter LE, Perry ST, Coggins L, Fuller FJ: Immune-mediated thrombocytopenia in horses infected with equine infectious anemia virus. J Virol 1991, 65:6242-5I

6. Reef VB, Dyson SS, Beech J: Lymphosarcoma and associated immune-mediated hemolytic anemia and thrombocytopenia in horses. J Am Vet Med Assoc 1984, 84:313-7

7. Cohen ND, Carter GK: Persistent thrombocytopenia in a case of equine infectious anemia. I Am Vet Med Assoc 199I, 1 99:750-2

8. Sellon DC, Walker KM, Russell KE, Perry ST, Covington P, Fuller FJ: Equine infectious anemia virus replication is upregulated during differentiation of blood monocytes from acutely infected horses. J Virol 1996, 70:590-4

9. Wardrop KJ, Baszler TV, Reilich E, Crawford TB: A morphometric study of bone marrow megakaryocytes in foals infected with equine infectious anemia virus. Vet Pathol 1996, 33:222-7

10. George JN: Platelet immunoglobulin G: its significance for the evaluation of thrombocytopenia and for understanding the origin of alpha-granule proteins. Blood 1990, 76:859-70

II. George JN, Saucerman S, Levine SP, Knieriem LK, Bainton DF: Immunoglobulin $\mathbf{G}$ is a platelet alpha granule-secreted protein. J Clin Invest 1985, 76:2020-5

12. George JN, Raskob GE: Idiopathic thrombocytopenic purpura: A concise summary of the pathophysiology and diagnosis in children and adults. Semin Hematol 1998, 35:5-8

13. George JN: measurement, interpretation, and clinical significance. Prog Hemost Thromb 1991, 10:97-126

14. Handagama PJ, Shuman MA, Bainton DF: The origin of platelet alpha-granule proteins. Prog Clin Biol Res 1990, 356:1 I9-30

15. Holme S, Heaton A, Kunchuba A, Hartman P: Increased levels of platelet associated IgG in patients with thrombocytopenia are not confined to any particular size class of platelets. $\mathrm{Br} J$ Haematol 1988, 68:431-6

16. Holme S, Heaton A, Konchuba A, Hartman P: Light scatter and total protein signal distribution of platelets by flow cytometry as parameters of size. J Lab Clin Med I988, I | 2:223-3|

17. Hughes M, Hayward CP, Warkentin TE, Horsewood P, Chorneyko KA, Kelton JG: Morphological analysis of microparticle generation in heparin-induced thrombocytopenia. Blood 2000, 96:188-94

18. Hughes M, Webert K, Kelton JG: The use of electron microscopy in the investigation of the ultrastructural morphology of immune thrombocytopenic purpura platelets [In Process Citation]. Semin Hematol 2000, 37:222-8

\section{Pre-publication history}

The pre-publication history for this paper can be accessed here:

http://www.biomedcentral.com/content/backmatter/ 1471-2326-1-1-b1.pdf
Publish with BioMedcentral and every scientist can read your work free of charge

"BioMedcentral will be the most significant development for disseminating the results of biomedical research in our lifetime." Paul Nurse, Director-General, Imperial Cancer Research Fund

Publish with BMc and your research papers will be:

- available free of charge to the entire biomedical community

- peer reviewed and published immediately upon acceptance

- cited in PubMed and archived on PubMed Central

- yours - you keep the copyright

Submit your manuscript here:

http://www.biomedcentral.com/manuscript/

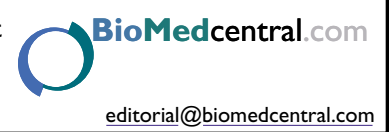

